PROCEEDINGS OF THE

AMERICAN MATHEMATICAL SOCIETY

Volume 135, Number 9, September 2007, Pages 2867-2876

S 0002-9939(07)08800-4

Article electronically published on May 8, 2007

\title{
A "DEFORMATION ESTIMATE" FOR THE TOEPLITZ OPERATORS ON HARMONIC BERGMAN SPACES
}

\author{
CONGWEN LIU
}

(Communicated by Joseph A. Ball)

Dedicated to Professor Jihuai Shi on the occasion of his seventieth birthday.

\begin{abstract}
Let $B$ denote the open unit ball in $\mathbb{R}^{n}$ for $n \geq 2$ and $d x$ the Lebesgue volume measure on $\mathbb{R}^{n}$. For $\alpha>-1$, the (weighted) harmonic Bergman space $b^{2, \alpha}(B)$ is the space of all harmonic functions $u$ which are in $L^{2}\left(B,\left(1-|x|^{2}\right)^{\alpha} d x\right)$. For $f \in L^{\infty}(B)$, the Toeplitz operator $T_{f}^{(\alpha)}$ is defined on $b^{2, \alpha}(B)$ by $T_{f}^{(\alpha)} u=Q_{\alpha}[f u]$, where $Q_{\alpha}$ is the orthogonal projection of $L^{2}\left(B,\left(1-|x|^{2}\right)^{\alpha} d x\right)$ onto $b^{2, \alpha}(B)$. In this note, we prove that for $f \in C(B) \cap L^{\infty}(B)$ radial, $\lim _{\alpha \rightarrow \infty}\left\|T_{f}^{(\alpha)}\right\|=\|f\|_{\infty}$.
\end{abstract}

\section{INTRODUCTION}

Let $B$ denote the open unit ball in $\mathbb{R}^{n}(n \geq 2)$ with boundary $S$. For $-1<\alpha<\infty$ let $V_{\alpha}$ be the measure on $B$ defined by

$$
d V_{\alpha}(x)=\frac{\Gamma(\alpha+n / 2+1)}{\pi^{n / 2} \Gamma(\alpha+1)}\left(1-|x|^{2}\right)^{\alpha} d x,
$$

where $d x$ denotes Lebesgue volume measure on $\mathbb{R}^{n}$. Clearly $V_{\alpha}$ is a probability measure on $B$. The harmonic Bergman space $b^{2, \alpha}(B)$ is the set of all harmonic functions $u$ which are in $L^{2}\left(B, V_{\alpha}\right)$. The space $b^{2, \alpha}(B)$ is in fact a closed subspace of $L^{2}\left(B, V_{\alpha}\right)$, thus a Hilbert space. We denote the orthogonal projection of $L^{2}\left(B, V_{\alpha}\right)$ onto $b^{2, \alpha}(B)$ by $Q_{\alpha}$. For $f \in L^{\infty}(B)$ the Toeplitz operator $T_{f}^{(\alpha)}: b^{2, \alpha}(B) \rightarrow b^{2, \alpha}(B)$ is defined by

$$
T_{f}^{(\alpha)} u=Q_{\alpha}[f u], \quad u \in b^{2, \alpha}(B) .
$$

The operator $T_{f}^{(\alpha)}$ is clearly bounded on $b^{2, \alpha}(B)$ and $\left\|T_{f}^{(\alpha)}\right\| \leq\|f\|_{\infty}$.

Our main result can be simply stated:

Theorem 1.1. Let $f \in C(B) \cap L^{\infty}(B)$ be radial. Then

$$
\lim _{\alpha \rightarrow \infty}\left\|T_{f}^{(\alpha)}\right\|=\|f\|_{\infty}
$$

Received by the editors November 30, 2005 and, in revised form, May 26, 2006.

2000 Mathematics Subject Classification. Primary 47B35, 47B38; Secondary 53D55.

Key words and phrases. Toeplitz operators, harmonic Bergman spaces, Deformation Estimate, Berezin type transforms.

This work was supported in part by the National Natural Science Foundation of China grant 10601025 . 
A result of this kind was first obtained for the Toeplitz operators $\mathbb{T}_{f}^{(\alpha)}$ on the holomorphic Bergman spaces on the unit disc by Klimek and Lesniewski [12. They even showed that the map $f \mapsto \mathbb{T}_{f}^{(\alpha)}$ satisfies "Rieffel's deformation estimates":

$$
\lim _{\alpha \rightarrow \infty}\left\|\mathbb{T}_{f}^{(\alpha)}\right\|=\|f\|_{\infty}
$$

for $f \in C(\bar{D})$ and

$$
\lim _{\alpha \rightarrow \infty}\left\|\alpha\left[\mathbb{T}_{f}^{(\alpha)}, \mathbb{T}_{g}^{(\alpha)}\right]-i \mathbb{T}_{\{f, g\}}^{(\alpha)}\right\|=0,
$$

for $f, g \in C^{\infty}(\bar{D})$, where $\left[\mathbb{T}_{f}^{(\alpha)}, \mathbb{T}_{g}^{(\alpha)}\right]=\mathbb{T}_{f}^{(\alpha)} \mathbb{T}_{g}^{(\alpha)}-\mathbb{T}_{g}^{(\alpha)} \mathbb{T}_{f}^{(\alpha)}$ and

$$
\{f, g\}(z):=i\left(1-|z|^{2}\right)^{2}[\partial f(z) \bar{\partial} g(z)-\bar{\partial} f(z) \partial g(z)]
$$

is the Poisson bracket of $f$ and $g$ corresponding to the Poincaré symplectic form on $D$. These estimates yield a quantum deformation of the unit disc in a sense close to Rieffel's "strict deformation quantization". The parameter $\alpha$ plays the role of the reciprocal of Planck's constant.

Similar results were also obtained by Coburn 7 in the contexts of the Fock spaces, by Borthwick, Lesniewski and Upmeier [6] for the weighted Bergman spaces on bounded symmetric domains in $\mathbb{C}^{n}$, by Bordemann, Meinrenken and Schlichenmaier [5] for the Hilbert spaces of holomorphic sections in the quantum line bundles over compact Kähler manifolds, and by Engliš [9] for the weighted Bergman space on smoothly bounded strictly pseudoconvex domains in $\mathbb{C}^{n}$.

All of the above referred results are about the Toeplitz operators on holomorphic spaces, since the standard Berezin and Berezin-Toeplitz quantizations on a Kähler manifold are based on weighted $L^{2}$-spaces of holomorphic functions. Recently, Engliš [10] explored the possibilities of using other function spaces instead, and he showed, in particular, that the Berezin-Toeplitz quantization based on harmonic Bergman spaces does not work, simply because the harmonic analogue of (1.3) fails. The main result of this note says that, however, (1.2) remains true in the harmonic Bergman spaces setting.

It should be noted that an extra radial assumption is made in Theorem 1.1, compared with the above referred results. For $n$ even this assumption can be dropped. In fact, in that case, by identifying $B$ and the unit ball of $\mathbb{C}^{n / 2}$ and denoting by $P^{(\alpha)}$ the projection in $b^{2, \alpha}$ onto the holomorphic Bergman space $L_{a}^{2}\left(B,\left(1-|z|^{2}\right)^{\alpha} d z\right)$, one has $\mathbb{T}_{f}^{(\alpha)}=P^{(\alpha)} T_{f}^{(\alpha)} P^{(\alpha)}$, whence $\|f\|_{\infty} \geq\left\|T_{f}^{(\alpha)}\right\| \geq\left\|\mathbb{T}_{f}^{(\alpha)}\right\|$. Thus (1.1) follows from its analytic analogue, for all bounded continuous symbols. However, this reasoning fails when $n$ is odd, and therefore we must take a different approach.

\section{Preliminaries}

Let $\mathcal{H}_{m}\left(\mathbb{R}^{n}\right)$ denote the space of all homogeneous harmonic polynomials on $\mathbb{R}^{n}$ of degree $m$. A spherical harmonic of degree $m$ is the restriction to $S$ of an element of $\mathcal{H}_{m}\left(\mathbb{R}^{n}\right)$. The collection of all spherical harmonics of degree $m$ will be denoted by $\mathcal{H}_{m}(S)$. Let $h_{m}$ denote the dimension of the vector space $\mathcal{H}_{m}(S)$. Although we will not use it, we remark that $h_{m}$ can be expressed in terms of binomial coefficients:

$$
h_{m}=\left(\begin{array}{c}
n+m-2 \\
n-2
\end{array}\right)+\left(\begin{array}{c}
n+m-3 \\
n-2
\end{array}\right)
$$


for $m \geq 1$. Every function in $b^{2, \alpha}(B)$ is a sum of homogeneous harmonic polynomials. For $m \geq 0$, let $Y_{m, j}, j=1, \ldots, h_{m}$, be an orthonormal basis for $\mathcal{H}_{m}(S)$. Then

$$
\bigcup_{m=0}^{\infty}\left\{p_{m, 1}^{(\alpha)}, \ldots, p_{m, h_{m}}^{(\alpha)}\right\}
$$

is an orthonormal basis for $b^{2, \alpha}(B)$, where

$$
p_{m, j}^{(\alpha)}(x)=\left\{\frac{\Gamma(\alpha+1+m+n / 2) \Gamma(n / 2)}{\Gamma(\alpha+1+n / 2) \Gamma(m+n / 2)}\right\}^{1 / 2}|x|^{m} Y_{m, j}(x /|x|), \quad x \in B .
$$

We need the following lemma whose proof can be found in [16, Lemma 4.2].

Lemma 2.1. If $f$ is a bounded measurable radial function on $B$, then each homogeneous harmonic polynomial of degree $m$ is an eigenvector of $T_{f}^{(\alpha)}$ with eigenvalue given by

$$
\lambda_{m}=\frac{2 \Gamma(m+n / 2+\alpha+1)}{\Gamma(m+n / 2) \Gamma(\alpha+1)} \int_{0}^{1} f^{*}(t)\left(1-t^{2}\right)^{\alpha} t^{2 m+n-1} d t,
$$

where $f^{*}$ is a bounded measurable function on $[0,1)$, for which $f(x)=f^{*}(|x|)$, for all $x \in B$.

Applying the above lemma to each $p_{m, j}^{(\alpha)}, j=1, \ldots, h_{m} ; m=0,1, \ldots$, we have

$$
\left\langle T_{f}^{(\alpha)} p_{m j}^{(\alpha)}, p_{m j}^{(\alpha)}\right\rangle=\lambda_{m}
$$

where $\langle\cdot, \cdot\rangle$ denotes the inner product in $b^{2, \alpha}(B)$. Hence

$$
\begin{aligned}
\int_{0}^{1} f^{*}(t) & \left(1-t^{2}\right)^{\alpha} t^{2 m+n-1} d t \\
& =\frac{\Gamma(m+n / 2) \Gamma(\alpha+1)}{2 h_{m} \Gamma(m+n / 2+\alpha+1)} \sum_{j=1}^{h_{m}}\left\langle T_{f}^{(\alpha)} p_{m j}^{(\alpha)}, p_{m j}^{(\alpha)}\right\rangle .
\end{aligned}
$$

We shall also make use of the Möbius transformations in several dimensions. We refer to 2 for the theory of these transformations.

Recall that the (full) Möbius group $\widehat{M}\left(\mathbb{R}^{n}\right)$ is the group generated by all similarities together with the reflection in the unit sphere

$$
x \mapsto x^{*}=\frac{x}{|x|^{2}} \quad\left(0^{*}=\infty, \infty^{*}=0\right) .
$$

We denote by $\widehat{M}(B)$ the subgroup which keeps the unit ball $B$ invariant. As in Ahlfors 2, for any $\psi \in \widehat{M}\left(\mathbb{R}^{n}\right)$, we denote by $\left|\psi^{\prime}(x)\right|$ the unique positive real number such that $\psi^{\prime}(x) /\left|\psi^{\prime}(x)\right| \in O(n)$. Here $O(n)$ denotes the orthogonal group and $\psi^{\prime}(x)$ the Jacobian matrix of $\psi$ at $x$.

Of particular interest is the Möbius transformation $\varphi_{a}$ defined for each $a \in B$ by

$$
\varphi_{a}(x)=\frac{|x-a|^{2} a-\left(1-|a|^{2}\right)(x-a)}{1-2 x \cdot a+|x|^{2}|a|^{2}},
$$

where $x \cdot a:=x_{1} a_{1}+\ldots+x_{n} a_{n}$ denotes the usual scalar product. $\varphi_{a}$ maps the unit ball $B$ onto itself, thereby sending the point $a$ into 0 . Actually the most general $\psi \in \widehat{M}(B)$ satisfying these conditions is of the form $K \varphi_{a}$, where $K \in O(n)$. We should remark that, although Ahlfors used the canonical mapping $T_{a}\left(=-\varphi_{a}\right)$ rather than $\varphi_{a}$, we find it more convenient to work with $\varphi_{a}$, since it is an involution. 
As we will see below, it does not matter for our purposes whether or not $\varphi_{a}$ is sensepreserving.

In the next lemma, we summarize the properties of the mapping $\varphi_{a}$ which will be used later. The proofs can be found in [2].

Lemma 2.2. For every $a \in B, \varphi_{a}$ has the following properties:

(1) $\varphi_{a}(0)=a$ and $\varphi_{a}(a)=0$.

(2) $\varphi_{a}$ is an involution, i.e., $\varphi_{a} \circ \varphi_{a}=I$, the identity mapping.

(3) The identity

$$
1-\left|\varphi_{a}(x)\right|^{2}=\frac{\left(1-|a|^{2}\right)\left(1-|x|^{2}\right)}{1-2 x \cdot a+|x|^{2}|a|^{2}}
$$

holds for every $x \in \bar{B}$.

(4) The identity

$$
\left|\varphi_{a}^{\prime}(x)\right|=\frac{1-|a|^{2}}{1-2 x \cdot a+|x|^{2}|a|^{2}}
$$

holds for every $x \in \bar{B}$.

(5) For any $\psi \in \widehat{M}(B)$ and $x \in \bar{B}$

$$
\varphi_{\psi(a)}(\psi(x))=\frac{\psi^{\prime}(a)}{\left|\psi^{\prime}(a)\right|} \varphi_{a}(x) .
$$

The following evaluation formula is also needed.

Lemma 2.3 ([14, Lemma 2.1]). For $x \in B$ and $\alpha \in \mathbb{C}$, we have

$$
\int_{S} \frac{d \sigma(\zeta)}{|x-\zeta|^{2 \alpha}}={ }_{2} F_{1}\left(\alpha, \alpha-\frac{n}{2}+1 ; \frac{n}{2} ;|x|^{2}\right) .
$$

Here ${ }_{2} F_{1}(\alpha, \beta ; \gamma ; z)$ denotes the Gauss hypergeometric function

$$
{ }_{2} F_{1}(\alpha, \beta ; \gamma ; z)=\sum_{k=0}^{\infty} \frac{(\alpha)_{k}(\beta)_{k}}{(\gamma)_{k}} \frac{z^{k}}{k !}
$$

with $\gamma \neq 0,-1,-2, \ldots$, where

$$
(\alpha)_{0}=1, \quad(\alpha)_{k}=\alpha(\alpha+1) \cdots(\alpha+k-1) \text { for } k \geq 1 .
$$

\section{BEREZIN TYPE TRANSFORMS}

In this section, we introduce a class of integral operators, called Berezin type transforms, which play an important role in the proof of Theorem 1.1. We believe that they are interesting in their own right and hope to study this kind of operator further in future publications.

For $\alpha>-1$ and $f \in L^{1}\left(B, d V_{\alpha}\right)$ define the Berezin-type transform $\mathscr{B}_{\alpha}$ of $f$ by

$$
\mathscr{B}_{\alpha} f(x)=\int_{B} f\left(\varphi_{x}(y)\right) j_{x}(y) d V_{\alpha}(y),
$$

where $\varphi_{x}$ is defined as in (2.4) and

$$
j_{x}(y):=\left(1-2 x \cdot y+|x|^{2}|y|^{2}\right)^{(2-n) / 2} .
$$

Replacing $y$ by $\varphi_{x}(y)$ in (3.1), we obtain a second formula for $\mathscr{B}_{\alpha} f$ :

$$
\mathscr{B}_{\alpha} f(x)=\int_{B} f(y) \frac{\left(1-|x|^{2}\right)^{\alpha+2}}{\left(1-2 x \cdot y+|x|^{2}|y|^{2}\right)^{\alpha+n / 2+1}} d V_{\alpha}(y) .
$$


Remark 1. In particular, if $n=2$, then (3.3) coincides with the original Berezin transform on the unit disk (see [4, p. 164], also [11, p. 29]).

Lemma 3.1. For any $x, y, a \in B$, we have

$$
j_{a}\left(\varphi_{y}(x) j_{y}(x)=j_{x}\left(\varphi_{y}(a)\right) j_{y}(a) .\right.
$$

Proof. Let us, for $x, y \in B$, introduce the temporary notation

$$
[x, y]:=\left(1-2 x \cdot y+|x|^{2}|y|^{2}\right)^{1 / 2} .
$$

Differentiation of (2.7) with respect to $x$ yields

$$
\varphi_{\psi(a)}^{\prime}(\psi(x)) \psi^{\prime}(x)=\frac{\psi^{\prime}(a)}{\left|\psi^{\prime}(a)\right|} \varphi_{a}^{\prime}(x) .
$$

Applying this with $\psi=\varphi_{y}$ and replacing $a$ with $\varphi_{y}(a)$, we have

$$
\left|\varphi_{a}^{\prime}\left(\varphi_{y}(x)\right)\right|\left|\varphi_{y}^{\prime}(x)\right|=\left|\varphi_{\varphi_{y}(a)}^{\prime}(x)\right| .
$$

In view of (2.6), this is just the same as

$$
\frac{1-|a|^{2}}{\left[\varphi_{y}(x), a\right]^{2}} \frac{1-|y|^{2}}{[x, y]^{2}}=\frac{1-\left|\varphi_{y}(a)\right|^{2}}{\left[x, \varphi_{y}(a)\right]^{2}} .
$$

Then, using (2.5), we finally obtain

$$
\left[\varphi_{y}(x), a\right]^{2}[x, y]^{2}=\left[x, \varphi_{y}(a)\right]^{2}[y, a]^{2},
$$

which gives (3.4).

Now we prove the "Möbius invariance" of the Berezin type transforms.

Proposition 3.2. Let $\alpha>-1$ and $a \in B$. Then

$$
\mathscr{B}_{\alpha}\left[\left(f \circ \varphi_{a}\right) j_{a}\right]=\left[\left(\mathscr{B}_{\alpha} f\right) \circ \varphi_{a}\right] j_{a}
$$

for every $f \in L^{1}\left(B, d V_{\alpha}\right)$.

Proof. For every $y \in B$, the Möbius transformation $\varphi_{\varphi_{a}(y)} \circ \varphi_{a} \circ \varphi_{y}$ fixes the origin, hence is some orthogonal transformation $K$. Recalling that $\varphi_{\varphi_{a}(y)}$ is an involution, we have

$$
\varphi_{a} \circ \varphi_{y}=\varphi_{\varphi_{a}(y)} \circ K
$$

Thus

$$
\begin{aligned}
\mathscr{B}_{\alpha}\left[\left(f \circ \varphi_{a}\right) j_{a}\right](y) & =\int_{B} f\left(\varphi_{a}\left(\varphi_{y}(x)\right)\right) j_{a}\left(\varphi_{y}(x)\right) j_{y}(x) d V_{\alpha}(x) \\
& \left.=\int_{B} f\left(\varphi_{\varphi_{a}(y)}(K x)\right) j_{a}\left(\varphi_{y}(x)\right) j_{y}(x) d V_{\alpha}(x) \quad \text { (by (3.6) }\right) \\
& \left.=j_{y}(a) \int_{B} f\left(\varphi_{\varphi_{a}(y)}(x)\right) j_{x}\left(K \varphi_{y}(a)\right) d V_{\alpha}(x) \quad \text { (by (3.4) }\right) \\
& =j_{a}(y) \int_{B} f\left(\varphi_{\varphi_{a}(y)}(x)\right) j_{\varphi_{a}(y)}(x) d V_{\alpha}(x) \\
& =\left(\mathscr{B}_{\alpha} f\right)\left(\varphi_{a}(y)\right) j_{a}(y),
\end{aligned}
$$

where in the second to the last equality we have used the fact that $K \varphi_{y}(a)=\varphi_{a}(y)$, which also follows from (3.6). 
Proposition 3.3. For any $f \in C(B) \cap L^{\infty}(B)$, we have

$$
\mathscr{B}_{\alpha} f(x) \rightarrow f(x), \quad x \in B,
$$

as $\alpha \rightarrow+\infty$.

Proof. It suffices to show that $\mathscr{B}_{\alpha} f(0) \rightarrow f(0)$ as $\alpha \rightarrow \infty$, since the general situation follows by applying it to $\left(f \circ \varphi_{x}\right) j_{x}$, in view of Proposition [3.2. Note that

$$
\mathscr{B}_{\alpha} f(0)-f(0)=\int_{B}[f(y)-f(0)] d V_{\alpha}(y) .
$$

The rest of the argument is standard. Splitting $B$ into a sufficiently small ball $\delta B$ and a spherical shell $B \backslash \delta B$ and correspondingly the integral, estimating the integral over $\delta B$ by the continuity of $f$, and estimating the integral over $B \backslash \delta B$ using the fact that $f$ is bounded and that

$$
\int_{B \backslash \delta B} d V_{\alpha}(y) \leq \frac{\Gamma(\alpha+1+n / 2)}{\Gamma(\alpha+1) \Gamma(n / 2+1)}\left(1-\delta^{2}\right)^{\alpha},
$$

we easily find that $\left(\mathscr{B}_{\alpha} f\right)(0) \rightarrow f(0)$ as $\alpha \rightarrow \infty$.

The following proposition gives the relation between the Berezin type transforms $\mathscr{B}_{\alpha}$ and the Toeplitz operators $T_{f}^{(\alpha)}$ on the harmonic Bergman spaces.

Proposition 3.4. Let $f \in L^{1}\left(B, d V_{\alpha}\right)$ be radial. We have

$$
\mathscr{B}_{\alpha} f(x)=\left(1-|x|^{2}\right)^{\alpha+2} \sum_{m=0}^{\infty} \frac{\Gamma(m+\alpha+2)}{\Gamma(\alpha+2) m ! h_{m}} \sum_{j=1}^{h_{m}}\left\langle T_{f}^{(\alpha)} p_{m j}^{(\alpha)}, p_{m j}^{(\alpha)}\right\rangle|x|^{2 m} .
$$

Proof. As in Lemma 2.1 we denote by $f^{*}$ the function on $[0,1)$, for which $f(x)=$ $f^{*}(|x|)$, for all $x \in B$. By integrating in polar coordinates $y=r \zeta$ and using (2.8), we see that

$$
\begin{aligned}
\mathscr{B}_{\alpha} f(x)= & \frac{n \Gamma(\alpha+1+n / 2)}{\Gamma(\alpha+1) \Gamma(n / 2+1)}\left(1-|x|^{2}\right)^{\alpha+2} \\
& \quad \times \int_{0}^{1}\left(\int_{S} \frac{d \sigma(\zeta)}{\left(1-2 r \zeta \cdot x+r^{2}|x|^{2}\right)^{\alpha+n / 2+1}}\right) f^{*}(r)\left(1-r^{2}\right)^{\alpha} r^{n-1} d r \\
= & \frac{n \Gamma(\alpha+1+n / 2)}{\Gamma(\alpha+1) \Gamma(n / 2+1)}\left(1-|x|^{2}\right)^{\alpha+2} \\
& \times \int_{0}^{1}{ }_{2} F_{1}\left(\frac{n}{2}+\alpha+1, \alpha+2 ; \frac{n}{2} ; r^{2}|x|^{2}\right) f^{*}(r)\left(1-r^{2}\right)^{\alpha} r^{n-1} d r \\
= & \left(1-|x|^{2}\right)^{\alpha+2} \sum_{m=0}^{\infty} \frac{2 \Gamma(m+n / 2+\alpha+1) \Gamma(m+\alpha+2)}{\Gamma(\alpha+1) \Gamma(\alpha+2) \Gamma(m+n / 2) m !} \\
& \times\left[\int_{0}^{1} f^{*}(t)\left(1-t^{2}\right)^{\alpha} t^{2 m+n-1} d t\right]|x|^{2 m} .
\end{aligned}
$$

This gives (5.4) after employing (2.3).

Remark 2. Korenblum and Zhu [13] proved that for a radial symbol the Toeplitz operator on the Bergman space of analytic functions on the unit disk is compact precisely when its Berezin transform vanishes at the unit circle. In [16 Stroethoff showed that the analogous result holds for Toeplitz operators on harmonic Bergman spaces. From (5.4), following the similar argument as in the proof of 16, Theorem 
4.1], one can show that the Berezin type transform $\mathscr{B}_{\alpha}$ also provide a compactness criterion for the Toeplitz operator: if $f$ is radial and is in $L^{\infty}(B)$, then $T_{f}^{(\alpha)}$ is compact if and only if $\mathscr{B}_{\alpha} f(x) \rightarrow 0$ as $|x| \rightarrow 1^{-}$.

\section{The Proof of Theorem 1.1}

Now we are ready to prove Theorem 1.1 . that

We note first that, for any $\alpha>-1,\left\|T_{f}^{(\alpha)}\right\| \leq\|f\|_{\infty}$. We must therefore prove

$$
\|f\|_{\infty} \leq \liminf _{\alpha \rightarrow \infty}\left\|T_{f}^{(\alpha)}\right\| .
$$

Note that

$$
\left|\left\langle T_{f}^{(\alpha)} p_{m j}^{(\alpha)}, p_{m j}^{(\alpha)}\right\rangle\right| \leq\left\|T_{f}^{(\alpha)}\right\|
$$

for each $p_{m j}^{(\alpha)}, j=1, \ldots, h_{m} ; m=0,1,2, \ldots$, and

$$
\sum_{m=0}^{\infty} \frac{\Gamma(m+\alpha+2)}{\Gamma(\alpha+2) m !}|x|^{2 m}=\frac{1}{\left(1-|x|^{2}\right)^{2+\alpha}} .
$$

It follows from (5.4) that for every $x \in B$,

$$
\left|\mathscr{B}_{\alpha} f(x)\right| \leq\left\|T_{f}^{(\alpha)}\right\| .
$$

From Proposition 3.3 it therefore follows that

$$
|f(x)|=\lim _{\alpha \rightarrow \infty}\left|\mathscr{B}_{\alpha} f(x)\right| \leq \liminf _{\alpha \rightarrow \infty}\left\|T_{f}^{(\alpha)}\right\| .
$$

As this holds for any $x \in B$, we get

$$
\|f\|_{\infty} \leq \liminf _{\alpha \rightarrow \infty}\left\|T_{f}^{(\alpha)}\right\|,
$$

and the theorem is proved.

\section{THE CASE $n=2$}

In this section, we shall prove Theorem 1.1 in the case of the unit disk, with the radial assumption removed. Although, as is mentioned in the introduction, this follows easily from its analytic analogue and a trivial observation, we give a direct proof. We feel that Proposition 5.1 below is of independent interest.

In two dimensions, it is convenient to use the complex coordinate, so we shall use different notation in this section. Let $D$ denote the open unit disk in the complex plane and $d A$ the normalized area measure on $D$. For $\alpha>-1$, the harmonic Bergman space $b^{2, \alpha}(D)$ is the set of all harmonic functions in $L^{2}\left(D, d A_{\alpha}\right)$, where

$$
d A_{\alpha}(z)=(\alpha+1)\left(1-|z|^{2}\right)^{\alpha} d A(z) .
$$

The orthogonal projection $Q_{\alpha}$ from $L^{2}\left(D, d A_{\alpha}\right)$ onto $b^{2, \alpha}(D)$ is given by

$$
Q_{\alpha}[f](z)=\int_{B} f(w) R_{\alpha}(z, w) d A_{\alpha}(w),
$$

where

$$
R_{\alpha}(z, w)=2 \operatorname{Re} \frac{1}{(1-z \bar{w})^{2+\alpha}}-1, \quad z, w \in D
$$


is the reproducing kernel of $b^{2, \alpha}(D)$ (see page 357 of [17]). Then we can write the Toeplitz operator $T_{f}^{(\alpha)}$ as an integral operator as follows:

$$
T_{f}^{(\alpha)} u(z)=\int_{D} R_{\alpha}(z, w) f(w) u(w) d A_{\alpha}(w) .
$$

Let $\mathfrak{L}\left(b^{2, \alpha}\right)$ be the algebra of all bounded linear operators on $b^{2, \alpha}(D)$. For any $T \in \mathfrak{L}\left(b^{2, \alpha}\right)$, the Berezin symbol (or the Berezin transform) of $T$ is the function $\widetilde{T}$ on $D$ defined by

$$
\widetilde{T}(z):=\frac{\left\langle T R_{\alpha}(\cdot, z), R_{\alpha}(\cdot, z)\right\rangle}{R_{\alpha}(z, z)} .
$$

By the Schwarz inequality we have $|\widetilde{T}(z)| \leq\|T\|$, hence $\widetilde{T}$ is actually a bounded function on $D$. In particular,

$$
\widetilde{T_{f}^{(\alpha)}}(z)=\int_{D} f(w) \frac{\left|R_{\alpha}(z, w)\right|^{2}}{R_{\alpha}(z, z)} d A_{\alpha}(w)
$$

and

$$
\left|\widetilde{T_{f}^{(\alpha)}}(z)\right| \leq\left\|T_{f}^{(\alpha)}\right\|
$$

for all $z \in D$.

Proposition 5.1. If $f \in C(D) \cap L^{\infty}(D)$, then

$$
\widetilde{T_{f}^{(\alpha)}}(z) \rightarrow f(z)
$$

as $\alpha \rightarrow \infty$.

Proof. Given $\varepsilon>0$, by uniform continuity, there is a $\delta=\delta(\varepsilon)$ so that $|f(w)-f(z)|<$ $\varepsilon / 2$ whenever $\left|\varphi_{z}(w)\right|<\delta$. Here $\varphi_{z}$ is the Möbius map interchanging 0 and $z$,

$$
\varphi_{z}(w)=\frac{z-w}{1-\bar{z} w}, \quad w \in D .
$$

Write

$$
\begin{aligned}
\widetilde{T_{f}^{(\alpha)}}(z)-f(z) & =\int_{D}[f(w)-f(z)] \frac{\left|R_{\alpha}(z, w)\right|^{2}}{R_{\alpha}(z, z)} d A_{\alpha}(w) \\
& =\int_{\left|\varphi_{z}(w)\right|<\delta}+\int_{\left|\varphi_{z}(w)\right| \geq \delta}=: I_{1}+I_{2} .
\end{aligned}
$$

We have

$$
\left|I_{1}\right| \leq \sup _{w \in \varphi_{z}(\delta D)}|f(w)-f(z)|<\varepsilon / 2
$$

and

Note that

$$
\left|I_{2}\right| \leq 2\|f\|_{\infty} \int_{\left|\varphi_{z}(w)\right| \geq \delta} \frac{\left|R_{\alpha}(z, w)\right|^{2}}{R_{\alpha}(z, z)} d A_{\alpha}(w) .
$$

and then

$$
R_{\alpha}(z, z)=\frac{2-\left(1-|z|^{2}\right)^{2+\alpha}}{\left(1-|z|^{2}\right)^{2+\alpha}} \geq \frac{1}{\left(1-|z|^{2}\right)^{2+\alpha}},
$$

$$
\begin{aligned}
\frac{\left|R_{\alpha}(z, w)\right|^{2}}{R_{\alpha}(z, z)} & \leq\left(1-|z|^{2}\right)^{2+\alpha}\left|2 \operatorname{Re} \frac{1}{(1-z \bar{w})^{2+\alpha}}-1\right|^{2} \\
& \leq 8 \frac{\left(1-|z|^{2}\right)^{2+\alpha}}{|1-z \bar{w}|^{4+2 \alpha}}+2\left(1-|z|^{2}\right)^{2+\alpha} .
\end{aligned}
$$


It is then enough to show that both integrals

$$
\int_{\left|\varphi_{z}(w)\right| \geq \delta} \frac{\left(1-|z|^{2}\right)^{2+\alpha}}{|1-z \bar{w}|^{4+2 \alpha}} d A_{\alpha}(w)
$$

and

$$
\left(1-|z|^{2}\right)^{2+\alpha} \int_{\left|\varphi_{z}(w)\right| \geq \delta} d A_{\alpha}(w)
$$

are less than $\varepsilon / 4$, for $\alpha$ sufficiently large.

Making the change of variables $w=\varphi_{z}(\eta)$ in (5.7), we have

$$
\int_{\left|\varphi_{z}(w)\right| \geq \delta} \frac{\left(1-|z|^{2}\right)^{2+\alpha}}{|1-z \bar{w}|^{4+2 \alpha}} d A_{\alpha}(w)=\int_{|\eta| \geq \delta} d A_{\alpha}(\eta) \leq(\alpha+1)\left(1-\delta^{2}\right)^{\alpha} .
$$

To estimate quantity (5.8), we note that for $z=0$ it reduces to (5.9); while for $z \neq 0$

$$
\left(1-|z|^{2}\right)^{2+\alpha} \int_{\left|\varphi_{z}(w)\right| \geq \delta} d A_{\alpha}(w) \leq\left(1-|z|^{2}\right)^{2+\alpha} \int_{D} d A_{\alpha}(w)=\left(1-|z|^{2}\right)^{2+\alpha} .
$$

The proof is complete.

Theorem 5.2. For any $f \in C(D) \cap L^{\infty}(D)$, we have

$$
\lim _{\alpha \rightarrow \infty}\left\|T_{f}^{(\alpha)}\right\|=\|f\|_{\infty}
$$

Proof. It follows from Proposition 5.1 and (5.4) that

$$
|f(z)|=\lim _{\alpha \rightarrow \infty}\left|\widetilde{T_{f}^{(\alpha)}}(z)\right| \leq \liminf _{\alpha \rightarrow \infty}\left\|T_{f}^{(\alpha)}\right\| .
$$

As this holds for any $z \in D$, we get

$$
\|f\|_{\infty} \leq \liminf _{\alpha \rightarrow \infty}\left\|T_{f}^{(\alpha)}\right\| .
$$

This, together with the obvious inequality $\left\|T_{f}^{(\alpha)}\right\| \leq\|f\|_{\infty}$, yields (5.10).

Remark 3. Proposition 5.1 and Theorem 5.2 can be extended to the pluriharmonic Bergman spaces $\mathfrak{b}^{2, \alpha}$, i.e., the subspace of $L^{2}\left(\mathbf{B}^{n},\left(1-|z|^{2}\right)^{\alpha} d z\right)$ consisting of all pluriharmonic functions on the unit ball $\mathbf{B}^{n}$ of $\mathbb{C}^{n}$, on which the Toeplitz operators and the Berezin transforms are defined likewise. In that setting, the reproducing kernels are given by $R_{\alpha}(z, w)=2 \operatorname{Re}(1-\langle z, w\rangle)^{-n-1-\alpha}-1$, and all arguments here go through unchanged.

Remark 4. At this point we can explain why the harmonic analogue of (1.3) fails.

The argument is taken from [10. It is a direct verification that $\widehat{T_{f}^{(\alpha)} T_{g}^{(\alpha)}}=\widehat{T_{g}^{(\alpha)} T_{f}^{(\alpha)}}$ for any $f, g \in L^{\infty}(D)$. (Note that the harmonic Bergman kernel $R_{\alpha}(x, y)$ is realvalued!) If (1.3) remains true with $T_{f}^{(\alpha)}$ in place of $\mathbb{T}_{f}^{(\alpha)}$, then, by taking the Berezin symbol and recalling that $|\widetilde{T}(x)| \leq\|T\|$ for any operator $T$ and any $x \in D$, we have

$$
\alpha\left(\widetilde{T_{f}^{(\alpha)} T_{g}^{(\alpha)}}-\widetilde{T_{g}^{(\alpha)} T_{f}^{(\alpha)}}\right)-\widetilde{i T_{\{f, g\}}^{(\alpha)}} \rightarrow 0
$$

as $\alpha \rightarrow \infty$, for any $f, g \in C^{\infty}(\bar{D})$. Thus

$$
\widetilde{T_{\{f, g\}}^{(\alpha)}} \rightarrow 0 \quad \text { as } \alpha \rightarrow \infty
$$


for any $f, g \in C^{\infty}(\bar{D})$. But by (5.5) we have $\widetilde{T_{\{f, g\}}^{(\alpha)}} \rightarrow\{f, g\}$ as $\alpha \rightarrow \infty$. This implies that $\{f, g\}=0$ for any $f, g \in C^{\infty}(\bar{D})$, which is absurd.

\section{ACKNOWLEDGMENT}

The author thanks the referee for his careful reading, valuable suggestions and corrections. The final version of the paper owes much to his constructive criticism.

\section{REFERENCES}

[1] P. Ahern, M. Flores, and W. Rudin. An invariant volume-mean-value property. J. Funct. Anal., 111(2):380-397, 1993. MR1203459 (94b:31002)

[2] L. V. Ahlfors. Möbius transformations in several dimensions. University of Minnesota, Minneapolis, Minn., 1981. MR.0725161 (84m:30028)

[3] S. Axler, P. Bourdon, and W. Ramey. Harmonic function theory, volume 137 of Graduate Texts in Mathematics. Springer-Verlag, New York, 2001. MR.1805196 (2001j:31001)

[4] F. A. Berezin. General concept of quantization. Comm. Math. Phys., 40:153-174, 1975. MR0411452 (53:15186)

[5] M. Bordemann, E. Meinrenken, and M. Schlichenmaier. Toeplitz quantization of Kähler manifolds and $\operatorname{gl}(N), N \rightarrow \infty$ limits. Comm. Math. Phys., 165(2):281-296, 1994. MR 1301849 (96f:58067)

[6] D. Borthwick, A. Lesniewski, and H. Upmeier. Nonperturbative deformation quantization of Cartan domains. J. Funct. Anal., 113(1):153-176, 1993. MR1214901 (94d:47065)

[7] L. A. Coburn. Deformation estimates for the Berezin-Toeplitz quantization. Comm. Math. Phys., 149(2):415-424, 1992. MR.1186036 (93j:47038)

[8] R. R. Coifman and R. Rochberg. Representation theorems for holomorphic and harmonic functions in $L^{p}$. In Representation theorems for Hardy spaces, volume 77 of Astérisque, pages 11-66. Soc. Math. France, Paris, 1980. MR0604369 (82j:32015)

[9] M. Engliš. Weighted Bergman kernels and quantization. Comm. Math. Phys., 227(2):211-241, 2002. MR.1903645 (2003f:32003)

[10] M. Engliš. Some variations on the Berezin quantization method. In: J. Govaerts, M.N. Hounkonnou, A.M. Msezane (editors), Proceedings of the Third Workshop on Contemporary Problems in Mathematical Physics (CoProMaPh3, Cotonou, Benin, November 2003), World Scientific, Singapore, 2004, pp. 450-464.

[11] H. Hedenmalm, B. Korenblum, and K. Zhu. Theory of Bergman spaces, Springer-Verlag, New York, 2000. MR1758653 (2001c:46043)

[12] S. Klimek and A. Lesniewski. Quantum Riemann surfaces. I. The unit disc. Comm. Math. Phys., 146(1):103-122, 1992. MR.1163670 (93g:58009)

[13] B. Korenblum and K. Zhu. An application of Tauberian theorems to Toeplitz operators. J. Operator Theory, 33(2):353-361, 1995. MR1354985 (96i:47046)

[14] C. Liu and L. Peng. Boundary regularity in the Dirichlet problem for the invariant Laplacians $\Delta_{\gamma}$ on the unit real ball. Proc. Amer. Math. Soc., 132(11):3259-3268 (electronic), 2004. MR2073300 (2005f:35057)

[15] E. M. Stein and G. Weiss. Introduction to Fourier analysis on Euclidean spaces. Princeton University Press, Princeton, N.J., 1971. MR0304972 (46:4102)

[16] K. Stroethoff. Compact Toeplitz operators on weighted harmonic Bergman spaces. J. Austral. Math. Soc. Ser. A, 64(1):136-148, 1998. MR1490152 (98k:47048)

[17] Z. Wu. Operators on harmonic Bergman spaces. Integral Equations Operator Theory, 24(3):352-371, 1996. MR1375980 (97c:47028)

Department of Mathematics, University of Science and Technology of China, Hefei, Anhui 230026 People's Republic of China

E-mail address: cwliu@nankai.edu.cn 